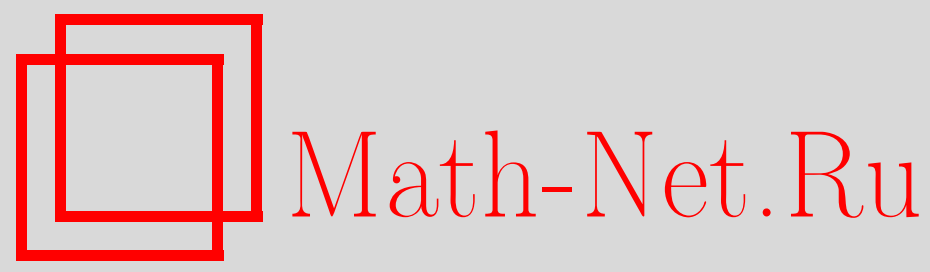

Н. И. Санду, Бесконечные независимые системы тождеств ассоциативной алгебры над бесконечным полем характеристики два, Матем. заметки, 2003, том 74, выпуск 4, 603611

DOI: https://doi.org/10.4213/mzm292

Использование Общероссийского математического портала Math-Net.Ru подразумевает, что вы прочитали и согласны с пользовательским соглашением http://www . mathnet.ru/rus/agreement

Параметры загрузки:

IP : 35.173 .137 .237

26 апреля 2023 г., 15:13:17

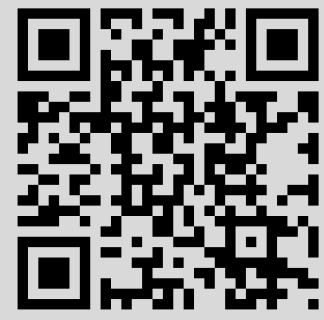




\section{БЕСКОНЕЧНЫЕ НЕЗАВИСИМЫЕ СИСТЕМЫ ТОЖДЕСТВ АССОЦИАТИВНОЙ АЛГЕБРЫ НАД БЕСКОНЕЧНЫМ ПОЛЕМ ХАРАКТЕРИСТИКИ ДВА}

\section{Н. И. Санду}

Пусть $\mathfrak{B}$ (соответственно $\mathfrak{D})$ означает многообразие ассоциативных (соответственно специальных йордановых) алгебр над бесконечным полем характеристики 2 , определенное тождеством $\left.\left(\left(\left(x_{1}, x_{2}\right), x_{3}\right),\left(\left(x_{4}, x_{5}\right), x_{6}\right)\right),\left(x_{7}, x_{8}\right)\right)=0$ (соответственно $\left.\left(\left(x_{1} x_{2} \cdot x_{3}\right)\left(x_{4} x_{5} \cdot x_{6}\right)\right)\left(x_{7} x_{8}\right)=0\right)$. В работе строятся бесконечные независимые системы тождеств в многообразии $\mathfrak{B}$ (соответственно в $\mathfrak{D})$. Отсюда выводится, что многообразие $\mathfrak{B}$ (соответственно $\mathfrak{D}$ ) содержит континуум различных не конечно базируемых подмногообразий и что в $\mathfrak{B}$ (соответственно в $\mathfrak{D}$ ) имеются алгебры с неразрешимой проблемой равенства слов.

Библиография: 16 названий.

В [1] Шпехтом (см. также [2, вопрос 1.164]) сфформулирована проблема о конечной базируемости всякого многообразия ассоциативных алгебр над полем характеристики нуль, которая совсем недавно положительно была решена Кемером [3]. Однако до сих пор не было известно, существуют ли не конечно базируемые многообразия ассоциативных алгебр? Как отмечается в [4], теперь в этом и состоит проблема Шпехта. Заметим, что в такой формулировке проблема Шпехта поставлена и Мальцевым в [5, вопрос 2.39].

В данной работе строятся неконечно базируемые многообразия ассоциативных алгебр над бесконечным полем $F$ характеристики 2 . Через $(a, b)=a b-b a$ обозначим коммутатор в алгебре, через $[a, b]=a^{-1} b^{-1} a b$-коммутатор в группе, через $b^{a}=a^{-1} b a$ - сопряжение элемента $b$ посредством элемента $a$ в группе. Будем использовать также запись

$$
\begin{aligned}
& \left(a_{1}, \ldots, a_{k-1}, a_{k}\right)=\left(\left(a_{1}, \ldots, a_{k-1}\right), a_{k}\right), \\
& {\left[a_{1}, \ldots, a_{k-1}, a_{k}\right]=\left[\left[a_{1}, \ldots, a_{k-1}\right], a_{k}\right] .}
\end{aligned}
$$

Пусть $\mathfrak{B}$ означает многообразие ассоциативных $F$-алгебр, определенное тождеством

$$
\left(\left(x_{1}, x_{2}, x_{3}\right),\left(x_{4}, x_{5}, x_{6}\right),\left(x_{7}, x_{8}\right)\right)=0 \text {, }
$$

$\mathfrak{C}$ (соответственно $\mathfrak{D})$ - многообразие лиевых $F$-алгебр (соответственно специальных йордановых $F$-алгебр), определенное тождеством

$$
\left(\left(x_{1} x_{2} \cdot x_{3}\right)\left(x_{4} x_{5} \cdot x_{6}\right)\right)\left(x_{7} x_{8}\right)=0
$$


Обозначим также

$$
\begin{aligned}
\mu_{k} & =\left((x, y, z),\left(x_{1}, x_{2}\right),\left(x_{3}, x_{4}\right), \ldots,\left(x_{2 k-1}, x_{2 k}\right),(x, y, z)\right) \\
\nu_{k} & \left.=\left(\ldots\left(\left(((x y) z)\left(x_{1} x_{2}\right)\right)\left(x_{3} x_{4}\right)\right) \ldots\left(x_{2 k-1} x_{2 k}\right)\right)((x y) z)\right) .
\end{aligned}
$$

Доказывается, что в многообразии $\mathfrak{B}$ система тождеств $\left\{\mu_{k}=0 \mid k=1,2, \ldots\right\}$ независима, т.е. никакое тождество этой системы не следует из остальных тождеств системы (теорема 1). Как следствие получается, что многообразие $\mathfrak{B}$ содержит континуум различных не конечно базируемых подмногообразий и что в $\mathfrak{B}$ существуют алгебры с неразрешимой проблемой равенства слов.

Из теоремы 1 также следует, что система тождеств $\left\{\nu_{k}=0 \mid k=1,2, \ldots\right\}$ независима в многообразиях $\mathfrak{C}$ и $\mathfrak{D}$. Этим получен отрищательный ответ на вопрос Слинько [2, вопрос 1.129] о конечной базируемости многообразия разрешимых йордановых алгебр в случае разрешимого индекса 4 многообразия специальных йордановых алгебр над бесконечным полем характеристики 2 .

Пусть $A$ - ассоциативная алгебра с единищей 1 и $B$ - ее подалгебра, удовлетворяющая тождеству

$$
x^{m}=0 .
$$

Тогда множество элементов $1-B=\{1-b \mid b \in B\}$ образуют группу и $(1-b)^{-1}=$ $1+b+b^{2}+\cdots+b^{m-1}$.

Лемма 1. Пусть $A$ - ассоциативная алгебра с единиией 1 и $B$ - ее подалгебра, удовлетворяющая тохсеству (3). Тогда для $u, v \in B$

$$
[1-u, 1-v]=1+\left(1+u+\cdots+u^{m-1}\right)\left(1+v+\cdots+v^{m-1}\right)(u, v) .
$$

ДоКАЗАТЕЛЬСТво. Имеем

$$
\begin{aligned}
& {[1-u, 1-v]=(1-u)^{-1}(1-v)^{-1}(1-u)(1-v)} \\
& \quad=(1-u)^{-1}(1-v)^{-1}(1-u)(1-v)-(1-u)^{-1}(1-v)^{-1}(1-v)(1-u)+1 \\
& \quad=1+(1-u)^{-1}(1-v)^{-1}((1-u)(1-v)-(1-v)(1-u)) \\
& \quad=1+(1-u)^{-1}(1-v)^{-1}(1-u, 1-v) \\
& \quad=1+(1-u)^{-1}(1-v)^{-1}(u, v) \\
& \quad=1+\left(1+u+\cdots+u^{m-1}\right)\left(1+v+\cdots+v^{m-1}\right)(u, v) .
\end{aligned}
$$

\section{Лемма доказана.}

Пусть $G$ - группа, $F$ - поле (ассоциативное коммутативное кольцо с единицей) и $F G$ групповая алгебра (групповое кольцо) группы $G$. Напомним, что $F G$ - это свободньй $F$-модуль с базисом $\{g \mid g \in G\}$ и произведение для элементов этого базиса определяется как их произведение в групше $G$. Если $H$ - подгруппа групшы $G$, то через $\omega H$ обозначим правьй идеал групповой алгебры $F G$, порожденньй множеством $\{1-h \mid h \in H\}$. Если $H=G$, то $\omega G$ назьвается фундаментальным идеалом групповой алгебры $F G$. 
Лемма 2 [6]. Пусть $H-$ nодгруппа группь $G$. Тогда

1) если әлементы $h_{i}$ порождают подгруппу $H$, то әлементы $1-h_{i}$ порождают правый идеал $\omega H$

2) $1-g \in \omega H$ тогда и только тогда, когда $g \in H$;

3) $Н$ является нормальной подгруппой в $G$ тогда и только тогда, когда $\omega H$ - двусторонний идеал алгебры $F G$;

4) если $H$ - нормальная подгруппа группы $G$, то $F(G / H)=F G / \omega H$;

5) $\omega G=\left\{\sum_{g \in G} \lambda_{g} g \mid \sum_{g \in G} \lambda_{g}=0\right\}$;

6) фундаментальный идеал $\omega G$ порождается как $F$-модуль әлементами вида $1-g, g \in G$.

ЛЕмма 3 [7]. Фундаментальный идеал $\omega G$ групповой алгебры $F G$ нильпотентен тогда и только тогда, когда $G$ - конечная р-группа и $F$ - поле характеристики $p$.

Рассмотрим теперь групшы $A_{n}, B_{n}$ и $C_{n}$, построенные в [8]. Группа $A_{n}$ имеет представление

$$
\left.A_{n}=\left\langle a_{1}, a_{2}, \ldots, a_{2 n}\right| a_{i}^{2}=1,\left[a_{i}, a_{j}, a_{k}\right]=1 \text { для всех } i, j, k\right\rangle .
$$

Она нильпотентна ступени 2 и имеет показатель 4 , а ее коммутант $A^{\prime}$ является элементарной абелевой 2 -группой. Если $u \in A^{\prime}$, то $u$ можно представить как произведение

$$
\prod_{1 \leqslant i<j \leqslant 2 n}\left[a_{i}, a_{j}\right]^{\beta_{i j}}
$$

где $\beta_{i j}=0 ; 1$. Пусть $\rho(u)$ означает сумму по модулю 2 коэффициентов одночленов

$$
x_{i_{1}} x_{i_{2}} x_{i_{3}} x_{i_{4}} \ldots x_{i_{2 n-1}} x_{i_{2 n}}
$$

где $\left\{i_{1}, i_{2}, \ldots, i_{2 n}\right\}=\{1,2, \ldots, 2 n\}$, в выражении

$$
\prod_{1 \leqslant i, j \leqslant 2 n}\left(1+x_{i j}\right)^{\beta_{i j}}
$$

Если $u \in A_{n}$, то через $\bar{u}$ обозначим образ $u$ при гомоморфизме $A_{n} \rightarrow A_{n} / A_{n}^{\prime}$.

Далее, $B_{n}=\left\langle b^{u}, c^{k} \mid u \in A_{n}, k \in A_{n} / A_{n}^{\prime}\right\rangle$, где $b \notin A_{n}$ и $B_{n}$ удовлетворяет соотношениям

$$
\begin{gathered}
\left(b^{u}\right)^{2}=\left(c^{k}\right)^{2}=1, \quad\left[b^{u}, b^{v}\right]=1, \quad \text { если } \bar{u} \neq \bar{v}, \\
{\left[b^{u}, b^{v}\right] c^{\bar{u} \rho\left(u v^{-1}\right)}, \quad \text { если } \bar{u}=\bar{v}, \quad\left[b^{u}, c^{k}\right]=1}
\end{gathered}
$$

для всех $u, v \in A_{n}, k \in A_{n} / A_{n}^{\prime}$.

Наконец, группа $C_{n}$ - это полупрямое произведение групшы $B_{n}$ и группы $A_{n}$. Из построения группы $C_{n}$ следует, что $C_{n}$ является конечной 2-группой. 
В группе $C_{n}$ верны тождества

$$
\begin{aligned}
\alpha & \left(x_{1}, x_{2}, \ldots, x_{8}\right)=\left[\left[x_{1}, x_{2}, x_{3}\right],\left[x_{4}, x_{5}, x_{6}\right],\left[x_{7}, x_{8}\right]\right]=1, \\
\beta_{k}= & \beta_{k}\left(x, y, z, u ; x_{1}, x_{2}, \ldots, x_{2 k}\right) \\
= & {\left[[x, y, z],\left[x_{1}, x_{2}\right],\left[x_{3}, x_{4}\right], \ldots,\left[x_{2 k-1}, x_{2 k}\right],[u, y, z]\right] } \\
& \times\left[[u, y, z],\left[x_{1}, x_{2}\right],\left[x_{3}, x_{4}\right], \ldots,\left[x_{2 k-1}, x_{2 k}\right],[x, y, z]\right]=1, \\
\gamma_{k}= & \gamma_{k}\left(x, y, z, u ; x_{1}, x_{2}, \ldots, x_{2 k}\right) \\
= & {\left[[x, y, z],\left[x_{1}, x_{2}\right],\left[x_{3}, x_{4}\right], \ldots,\left[x_{2 k-1}, x_{2 k}\right],[x, y, u]\right] } \\
& \times\left[[x, y, u],\left[x_{1}, x_{2}\right],\left[x_{3}, x_{4}\right], \ldots,\left[x_{2 k-1}, x_{2 k}\right],[x, y, z]\right]=1, \\
\delta_{k}= & \delta_{k}\left(x, y, z ; x_{1}, x_{2}, \ldots, x_{2 k}\right) \\
= & {\left[[x, y, z],\left[x_{1}, x_{2}\right],\left[x_{3}, x_{4}\right], \ldots,\left[x_{2 k-1}, x_{2 k}\right],[x, y, z]\right]=1, }
\end{aligned}
$$

если $k \neq n$, и верно неравенство

$$
\left[\left[b, a_{1}, a_{2}\right],\left[a_{1}, a_{2}\right],\left[a_{3}, a_{4}\right], \ldots,\left[a_{2 n-1}, a_{2 n}\right],\left[b, a_{1}, a_{2}\right]\right] \neq 1 .
$$

Тождества (4), (7) и неравенство (8) доказаны в [8]. По построению $C_{n} / B_{n}=A_{n}$ и группа $A_{n}$ нильпотентна ступени 2 . Поэтому для $x, y, z \in C_{n}$ имеем $[x, y, z] \in B_{n}$. При доказательстве леммы 2 из [8] показано, что для $p_{1}, p_{2} \in B_{n}, u_{1}, u_{2}, \ldots, u_{k} \in A_{n}$ верно равенство $\left[p_{1}, u_{1}, u_{2}, \ldots, u_{k}, p_{2}\right]=\left[p_{2}, u_{1}, u_{2}, \ldots, u_{k}, p_{1}\right]$. Подгрупша $B_{n}$ нормальна в группе $C_{n}$. Тогда $\left[p_{2}, u_{1}, u_{2}, \ldots, u_{k}\right] \in B_{n}$ и из определения группы $B_{n}$ следует, что $\left[p_{2}, u_{1}, u_{2}, \ldots, u_{k}, p_{1}\right]^{2}=1$. Тогда, используя эти равенства, аналогично доказательству тождества (9) из [8] показьвается, что в групе $C_{n}$ верны и тождества $(5),(6)$. Из (4)-(8) вытекает

ЛЕмма 4. В многообразии групп тождество $\delta_{n}=1$ не является следствием системы тождеств $\beta_{k}=1, \gamma_{k}=1, \delta_{k}=1$ при $k \neq n u \alpha\left(x_{1}, x_{2}, \ldots, x_{8}\right)=1$.

В дальнейшем будем считать, что $F$ - бесконечноеполе характеристики 2 . Группа $C_{n}$ является конечной 2-группой, поэтому из леммы 3 следует, что фундаментальньй идеал $\omega C_{n}$ нильпотентен. Тогда, как показано перед леммой 1, множество $R_{n}=1-\omega C_{n}$ образуют группу. Очевидно, что $C_{n} \subseteq R_{n}$, поэтому $F C_{n} \subseteq F R_{n}$ и $\omega C_{n} \subseteq \omega R_{n}$. Но, используя п. 6) леммы 2 , легко показать, что $\omega C_{n}=\omega R_{n}$. Через $r$ обозначим элементы группы $R_{n}$, и пусть $K$ - подгруппа группы $R_{n}$, порожденная всеми выражениями

$$
\begin{gathered}
\alpha\left(r_{1}, r_{2}, \ldots, r_{8}\right), \quad \beta_{k}\left(r_{i}, r_{j}, r_{s}, r_{t} ; r_{1}, r_{2}, \ldots, r_{2 k}\right), \\
\gamma_{k}\left(r_{i}, r_{j}, r_{s}, r_{t} ; r_{1}, r_{2}, \ldots, r_{2 k}\right), \quad \delta_{k}\left(r_{i}, r_{j}, r_{s} ; r_{1}, r_{2}, \ldots, r_{2 k}\right)
\end{gathered}
$$

при $k \neq n$. Очевидно, что подгруппа $K$ нормальна в $R_{n}$ и что в $R_{n} / K$ верны тождества (4)-(7). Через $d, g_{i}$ обозначим образы элементов $b, a_{i}$ при гомоморфизме $R_{n} \rightarrow R_{n} / K=T_{n}$

По лемме 4 тождество $\delta_{n}=1$ не является следствием системы тождеств (4)-(7). Тогда из (8) следует, что в группе $T_{n}$ верно неравенство

$$
\delta_{n}\left(d, g_{1}, g_{2} ; g_{1}, g_{2}, \ldots, g_{2 k}\right) \neq 1 \text {. }
$$

Подгруппа $K$ нормальна в $R_{n}$. Тогда по п. 3) леммы $2 \omega K$ будет идеалом алгебры $\omega R_{n}$. Из п. 5) леммы 2 следует, что гомоморфизм $F R_{n} \rightarrow F R_{n} / \omega K$ coxраняет сумму 
коэффициентов. Поэтому из соотношения $F\left(R_{n} / K\right) \cong F R_{n} / \omega K$ (п. 5) леммы 2 ) следует, что $\omega\left(R_{n} / K\right) \cong \omega R_{n} / \omega K$. Но $\omega R_{n}=\omega C_{n}$, и алгебра $\omega C_{n}$ нильпотентна. Тогда и алгебра $\omega\left(R_{n} / K\right)=\omega T_{n}$ нильпотентна, например, индекса $m$. Через $u_{i}$ в дальнейшем будем обозначать элементы группы $C_{n}$, а через $v_{i}$ - образ элементов $u_{i}$ при гомоморфизме $R_{n} \rightarrow R_{n} / K$. Пусть $\alpha_{i} \in F$. Введем обозначения

$$
\begin{gathered}
r_{i}=1-\alpha_{i} w_{i}, \quad w_{i}=1-v_{i}, \\
\{x, y\}=-\left(1+x+\cdots+x^{m-1}\right)\left(1+y+\cdots+y^{m-1}\right)(x, y), \\
\left\{x_{1}, \ldots, x_{i-1}, x_{i}\right\}=\left\{\left\{x_{1}, \ldots, x_{i-1}\right\}, x_{i}\right\} .
\end{gathered}
$$

Из леммы 1 следует, что $\left[r_{i}, r_{j}\right]=1-\left\{\alpha_{i} w_{i}, \alpha_{j} w_{j}\right\}$, а отсюда непосредственно получаем, что

$$
\left[r_{1}, r_{2}, \ldots, r_{k}\right]=1-\left\{\alpha_{1} w_{1}, \alpha_{2} w_{2}, \ldots, \alpha_{k} w_{k}\right\}
$$

Обозначим также

$$
\begin{aligned}
& \theta\left(x_{1}, x_{2}, \ldots, x_{8}\right)=\left\{\left\{x_{1}, x_{2}, x_{3}\right\},\left\{x_{4}, x_{5}, x_{6}\right\},\left\{x_{7}, x_{8}\right\}\right\}, \\
& \eta_{k}\left(x_{s}, x_{t}, x_{i}, x_{j} ; x_{1}, x_{2}, \ldots, x_{2 k}\right) \\
& =\left\{\left\{x_{s}, x_{i}, x_{j}\right\},\left\{x_{1}, x_{2}\right\}, \ldots,\left\{x_{2 k-1}, x_{2 k}\right\},\left\{x_{t}, x_{i}, x_{j}\right\}\right\} \\
& -\left\{\left\{x_{t}, x_{i}, x_{j}\right\},\left\{\left\{x_{s}, x_{i}, x_{j}\right\},\left\{x_{1}, x_{2}\right\}, \ldots,\left\{x_{2 k-1}, x_{2 k}\right\}\right\}\right\}, \\
& \xi_{k}\left(x_{s}, x_{t}, x_{i}, x_{j} ; x_{1}, x_{2}, \ldots, x_{2 k}\right) \\
& =\left\{\left\{x_{i}, x_{j}, x_{s}\right\},\left\{x_{1}, x_{2}\right\}, \ldots,\left\{x_{2 k-1}, x_{2 k}\right\},\left\{x_{i}, x_{j}, x_{t}\right\}\right\} \\
& -\left\{\left\{x_{i}, x_{j}, x_{t}\right\},\left\{\left\{x_{i}, x_{j}, x_{s}\right\},\left\{x_{1}, x_{2}\right\}, \ldots,\left\{x_{2 k-1}, x_{2 k}\right\}\right\}\right\}, \\
& \lambda_{k}\left(x_{i}, x_{j}, x_{s} ; x_{1}, x_{2}, \ldots, x_{2 k}\right) \\
& =\left\{\left\{x_{i}, x_{j}, x_{s}\right\},\left\{x_{1}, x_{2}\right\}, \ldots,\left\{x_{2 k-1}, x_{2 k}\right\},\left\{x_{i}, x_{j}, x_{s}\right\}\right\} .
\end{aligned}
$$

Теперь покажем, что в алгебре $\omega T_{n}$ верны равенства

$$
\begin{aligned}
\theta & =\theta\left(\alpha_{1} w_{1}, \alpha_{2} w_{2}, \ldots, \alpha_{8} w_{8}\right)=0 \\
\eta_{k} & =\eta_{k}\left(\alpha_{s} w_{s}, \alpha_{t} w_{t}, \alpha_{i} w_{i}, \alpha_{j} w_{j} ; \alpha_{1} w_{1}, \alpha_{2} w_{2}, \ldots, \alpha_{2 k} w_{2 k}\right)=0, \\
\xi_{k} & =\xi_{k}\left(\alpha_{s} w_{s}, \alpha_{t} w_{t}, \alpha_{i} w_{i}, \alpha_{j} w_{j} ; \alpha_{1} w_{1}, \alpha_{2} w_{2}, \ldots, \alpha_{2 k} w_{2 k}\right)=0 \\
\lambda_{k} & =\lambda_{k}\left(\alpha_{i} w_{i}, \alpha_{j} w_{j}, \alpha_{s} w_{s} ; \alpha_{1} w_{1}, \alpha_{2} w_{2}, \ldots, \alpha_{2 w} v_{2 k}\right)=0 .
\end{aligned}
$$

Действительно, подставляем в $(9) r_{i}=1-\alpha_{i}\left(1-u_{i}\right), \alpha_{i} \in F$, и пусть образ полученного выражения для $\beta_{k}\left(r_{i}, r_{j}, r_{s}, r_{t} ; r_{1}, r_{2}, \ldots, r_{2 k}\right)$ при гомоморфизме $R_{n} \rightarrow R_{n} / K$ имеет вид $\phi_{k} \rho_{k}$. Тогда в групе $R_{n} / K$ верно равенство $\phi_{k} \rho_{k}=1$ или $\phi_{k}=\rho_{k}^{-1}$. Здесь $\phi_{k}, \rho_{k}$ - коммутаторные выражения групшы $T_{n}$. При помоши тождества $[u, v]=[v, u]^{-1}$ представим $\rho_{k}^{-1}$ в виде $\psi_{k}$, в котором расположение квадратных скобок в $\psi_{k}$ совпадает с расположением фигурных скобок во втором члене выражения $\eta_{k}$. Расположения скобок в $\phi_{k}$ и в первом члене из $\eta_{k}$ совпадают. Теперь для $\phi_{k}, \psi_{k}$ применяем равенство (11). Предположим, что $\phi_{k}=1-\bar{\phi}_{k}, \psi=1-\bar{\psi}_{k}$. Так как в группе $R_{n} / K$ верно равенство $\phi_{k}=\psi_{k}$, то из соотношения $\omega\left(R_{n} / K\right) \cong \omega R_{n} / \omega K$ следует, что в алгебре $\omega T_{n}$ верно равенство $\bar{\phi}_{k}-\bar{\psi}_{k}=0$. Но $\bar{\phi}_{k}-\bar{\psi}_{k}=\eta_{k}$. Поэтому в алгебре $\omega T_{n}$ верно равенство $\eta_{k}=0$. Аналогично показывается, что в $\omega T_{n}$ верны равенства $\theta=0, \xi_{k}=0, \lambda_{k}=0$.

Пусть теперь $f=f\left(x_{1}, x_{2}, \ldots, x_{t}\right)$ - один из многочленов

$$
\begin{gathered}
\theta\left(x_{1}, x_{2}, \ldots, x_{8}\right), \quad \eta_{k}\left(x_{s}, x_{t}, x_{i}, x_{j} ; x_{1}, x_{2}, \ldots, x_{2 k}\right), \\
\xi_{k}\left(x_{s}, x_{t}, x_{i}, x_{j} ; x_{1}, x_{2}, \ldots, x_{2 k}\right), \quad \lambda_{k}\left(x_{i}, x_{j}, x_{s} ; x_{1}, x_{2}, \ldots, x_{2 k}\right) .
\end{gathered}
$$


Переходим согласно определению операции $\{\cdot, \cdot\}$ в $f$ к операциям,$+ \cdot$ и для полученного многочлена естественным образом вводим понятия степени по каждой из переменных $x_{i}$, степени и однородности многочлена. Представим $f$ в виде $f=f_{0}+f_{1}+\cdots+f_{r_{1}}$, где $f_{i}$ - сумма всех одночленов многочлена $f$, имеющих степень $i$ по $x_{1}$. Пусть $w_{1}, w_{2}, \ldots, w_{t}$ - элементы алгебры $\omega T_{n}$, определенные выше. Будем сокрашенно обозначать $f\left(w_{1}, w_{2}, \ldots, w_{t}\right)$ через $f(w)$. Если $\alpha \in F$, то

$$
f\left(\alpha w_{1}, w_{2}, \ldots, w_{t}\right)=\alpha^{0} f_{0}(w)+\alpha^{1} f_{1}(w)+\cdots+\alpha^{r_{1}} f_{r_{1}}(w) .
$$

Пусть $\alpha_{1}, \alpha_{2}, \ldots, \alpha_{r_{1}}$ - произвольные элементы из $F$. Тогда из $(12)$ получим систему уравнений

$$
\alpha_{i}^{0} f_{0}(w)+\alpha_{i}^{1} f_{1}+\cdots+\alpha_{i}^{r_{1}} f_{r_{1}}(w)=0
$$

от переменных $f_{0}(w), f_{1}(w), \ldots, f_{r_{1}}(w)$. По [9, c. 376] $d f_{i}(w)=0$, где $d$ - определитель, составленньй из строчек $\alpha_{i}^{0}, \alpha_{i}^{1}, \ldots, \alpha_{i}^{r_{1}}$. Поле $F$ бесконечное. Поэтому можно выбрать $\alpha_{1}, \alpha_{2}, \ldots, \alpha_{r_{1}}$ так, чтобы $d \neq 0$. Поэтому $f_{i}(w)=0$. Проделав ту же операцию с многочленами $f_{i}, i=0,1, \ldots, r_{1}$, и переменной $x_{2}$ и т. д., в конце конщов получим следующее утверждение.

ЛЕмма 5. Пусть

$$
f=f_{1}\left(x_{1}, x_{2}, \ldots, x_{t}\right)+\cdots+f_{i}\left(x_{1}, x_{2}, \ldots, x_{t}\right)+\cdots+f_{r}\left(x_{1}, x_{2}, \ldots, x_{t}\right)
$$

- разлохсение многочлена $f$ на однородные компоненты $f_{i}\left(x_{1}, x_{2}, \ldots, x_{t}\right)$, и пусть $w_{1}, w_{2}, \ldots, w_{t}$ - әлементы алгебры $\omega T_{n}$, определенные выше. Тогда

$$
f_{i}\left(w_{1}, w_{2}, \ldots, w_{t}\right)=0
$$

В частности, рассматривая однородные компоненты наименьшей степени, получим, что в алгебре $\omega T_{n}$ при $k \neq n$ верны равенства

$$
\begin{gathered}
\left(\left(w_{1}, w_{2}, w_{3}\right),\left(w_{4}, w_{5}, w_{6}\right),\left(w_{7}, w_{8}\right)\right)=0 \\
\left(\left(w_{i}, w_{j}, w_{s}\right),\left(w_{1}, w_{2}\right), \ldots,\left(w_{2 k-1}, w_{2 k}\right),\left(w_{t}, w_{j}, w_{s}\right)\right) \\
-\left(\left(w_{i}, w_{j}, w_{s}\right),\left(\left(w_{t}, w_{j}, w_{s}\right),\left(w_{1}, w_{2}\right), \ldots,\left(w_{2 k-1}, w_{2 k}\right)\right)\right)=0 \\
\left(\left(w_{i}, w_{j}, w_{s}\right),\left(w_{1}, w_{2}\right), \ldots,\left(w_{2 k-1}, w_{2 k}\right),\left(w_{i}, w_{j}, w_{t}\right)\right) \\
-\left(\left(w_{i}, w_{j}, w_{s}\right),\left(\left(w_{i}, w_{j}, w_{t}\right),\left(w_{1}, w_{2}\right), \ldots,\left(w_{2 k-1}, w_{2 k}\right)\right)\right)=0 \\
\left(\left(w_{i}, w_{j}, w_{s}\right),\left(w_{1}, w_{2}\right), \ldots,\left(w_{2 k-1}, w_{2 k}\right),\left(w_{i}, w_{j}, w_{s}\right)\right)=0
\end{gathered}
$$

Согласно п. 6) леммы 2 фундаментальньй идеал $\omega C_{n}$ порождается как $F$-модуль элементами вида $v_{i}=1-u_{i}$. Поэтому $F$-модуль $\omega T_{n}$ порождается элементами вида $w_{i}$, т.е. любой элемент $h \in \omega T_{n}$ имеет разложение $h=\alpha_{1} w_{1}+\cdots+\alpha_{s} w_{s}$. Тогда при помощи последних равенств, используя тождество $(x, y)=-(y, x)$, индукцией по длине $s$, легко доказать следующее утверждение.

Лемма 6. В алгебре $\omega T_{n}$ верны тождества (1) $u \mu_{k}=0$ при $k \neq n$.

Лемма 7. В фундаментальном идеале $\omega T_{n}$ групповой алгебры $F T_{n}$ тождество $\mu_{n}=0$ не верно. 
ДокАЗАТЕльство. Пусть $A(Z, r)$ - свободная ассоциативная алгебра над кольцом целых чисел $Z$ от множества свободных порождающих $x_{1}, x_{2}, \ldots, x_{r}$. По тереме 5.6 из [10] элементы $y_{i}=1-x_{i}$ порождают свободную грушу $S$ и $y_{i}$ являются свободными порождаюшими. (В теореме 5.6 рассматриваются элементы вида $1+x_{i}$, однако теорема верна, если рассмотреть и элементы вида $1-x_{i}$ ). Очевидно, что относительно коммутирования $(u, v)=u v-v u$ порождающие элементы произвольной ассоциативной алгебры $B$ над кольцом $Q$ порождают алгебру Ли $L(Q, B)$. В частности, из теоремы 5.9 из [10] следует, что если рассмотреть алгебру $A=A(Z, r)$, то $L(Z, A)$ будет свободной алгеброй Ли над кольцом $Z$ с множеством свободных порождающих $x_{1}, x_{2}, \ldots, x_{r}$.

Обозначим через $G$ подгруппу групшы $T_{n}$, порож денную элементами $d, g_{1}, g_{2}, \ldots, g_{2 n}$, а через $\Omega(Z, G)=\Omega(G)$ - подалгебру группового кольца $Z G$, порожденную элементами

$$
\bar{d}=1-d, \quad \bar{g}_{i}=1-g_{i}, \quad i=1,2, \ldots, 2 n .
$$

Пусть $r=2 n+1$. Алгебра $A(Z, r)$ свободна со свободными порождающими $x_{1}, x_{2}, \ldots$, $x_{2 n+1}$. Тогда отображения

$$
x_{2 n+1} \rightarrow \bar{d}, \quad x_{i} \rightarrow \bar{g}_{i}, \quad i=1,2, \ldots, 2 n
$$

определяют эпиморфизм алгебр $A(Z, r) \rightarrow \Omega(Z, G)$. В частности, они определяют эпиморфизм алгебр Ли $L(Z, A) \rightarrow L(Z, \Omega G)$ и эпиморфизм групп $S \rightarrow G$, при котором

$$
y_{2 n+1} \rightarrow d, \quad y_{i} \rightarrow g_{i}, \quad i=1,2, \ldots, 2 n
$$

Пусть $G=S / H$. Через $S_{i}, G_{i}$ соответственно обозначим члены нижних центральных рядов групп $S$ и $G$, через $L_{i}(Z, A)$ - подмодуль свободной алгебры Ли $L(Z, A)$, состоящий из всех однородных элементов степени $i$, а через $L_{i}(Z, \Omega G)$ - образ модуля $L_{i}(Z, A)$ при эпиморфизме $A(Z, r) \rightarrow \Omega(Z, G)$. Пусть $\beta^{i}\left(x_{j_{1}}, \ldots, x_{j_{i}}\right)$ - элемент из $L_{i}(Z, A)$. По теореме 5.12 из [10] отображение

$$
\beta^{i}\left(y_{j_{1}}, \ldots, y_{j_{i}}\right) S_{i+1} \rightarrow \beta^{i}\left(x_{j_{1}}, \ldots, x_{j_{i}}\right)
$$

является изоморфизмом фактор-групшы $S_{i} / S_{i+1}$ на аддитивную группу $L_{i}(Z, A)$; при этом расположение скобок $\beta^{i}$ означает коммутирование в группе $S$ и умножение в алгебре $L(Z, A)$. Далее, при эпиморфизме $S \rightarrow S / H$ подгруппы $S_{i}$ и $S_{i+1}$ отображаются на $S_{i} H / H$ и $S_{i+1} H / H$ соответственно. Тогда по теоремам о гомоморфизмах получаем

$$
\begin{aligned}
G_{i} / G_{i+1} & =\left(S_{i} H / H\right) /\left(S_{i+1} H / H\right) \cong S_{i} H / S_{i+1} H \\
& \cong S_{i} /\left(S_{i} \cap H\right) S_{i+1} \cong\left(S_{i} / S_{i+1}\right) /\left(\left(S_{i} \cap H\right) S_{i+1} / S_{i+1}\right) .
\end{aligned}
$$

Это означает ввиду (13), что эпиморфизм $A(Z, r) \rightarrow \Omega(Z, G)$ индуцирует эпиморфизм модуля $L_{i}(Z, A)$ на модуль $L_{i}(Z, \Omega G)$, ядро $M_{i}$ которого изоморфно подмодулю из $S_{i} / S_{i+1}$, состоящему из тех смежных классов по $S_{i+1}$, которые определяются элементами из $S_{i} \cap H$. Далее, из неравенства (8) следует, что $\delta_{n}\left(y_{2 n+1}, y_{1}, y_{2} ; y_{1}, y_{2}, \ldots, y_{2 n}\right) \notin H$. Тогда

$$
\mu_{n}\left(x_{2 n+1}, x_{1}, x_{2} ; x_{1}, x_{2}, \ldots, x_{2 n}\right) \notin M_{2 n+6},
$$


а так как $L_{2 n+6}(Z, A) / M_{2 n+6} \cong L_{2 n+6}(Z, \Omega G)$, то отсюда следует, что в алгебре $L(Z, \Omega G)$ верно неравенство

$$
\mu_{n}\left(\bar{d}, \bar{g}_{1}, \bar{g}_{2} ; \bar{g}_{1}, \bar{g}_{2}, \ldots, \bar{g}_{2 n}\right) \neq 0
$$

В группе $C_{n}$ вьполнены равенства $b^{2}=1, a_{i}^{2}=1$, которые индуцируют равенства $d^{2}=1, g_{i}^{2}=1$ в групе $G$. Тогда из изоморфизма модулей $G_{i} / G_{i+1} \cong L_{i}(Z, \Omega G)$, указанного в $(14)$, следует, что в алгебре $L(Z, \Omega G)$ верны равенства $2 \bar{d}=0,2 \bar{g}_{i}=0$. Алгебры $L(Z, \Omega G), \Omega(Z, G)$ порождаются элементами $\bar{d}, \bar{g}_{i}$. Тогда они являются алгебрами над кольцом $Z_{2}$ классов вычетов по модулю 2. Очевидно, что $Z_{2}$ является подполем бесконечного поля $F$ характеристики 2. Рассмотрим свободные ассоциативные алгебры $A\left(Z_{2}, r\right)$ и $A(Z, r)$ от множества свободных порождающих $x_{1}, x_{2}, \ldots, x_{2 n+1}$. Алгебра $A\left(Z_{2}, r\right)$ является подалгеброй алгебры $A(Z, r)$, так как обе они обладают одним и тем же линейным базисом одночленов. Более того, по теореме Витта $\left[11\right.$, c. 187] $L\left(Z_{2}, A\right)$ и $L(F, A)$ являются свободными алгебрами Ли от множества свободных порождающих $x_{1}, x_{2}, \ldots, x_{2 n+1}$ и согласно [12] они обладают одним и тем же линейным базисом, состоящим из слов от переменных $x_{1}, x_{2}, \ldots, x_{2 n+1}$. Поэтому и алгебра $L\left(Z_{2}, A\right)$ является подалгеброй алгебры $L(F, A)$. Далее, при помощи отображений

$$
x_{2 n+1} \rightarrow \bar{d}, \quad x_{i} \rightarrow \bar{g}_{i}, \quad i=1,2, \ldots, 2 n,
$$

как и в случае кольца $Z$, определим алгебры $\left.\Omega\left(Z_{2}, G\right), L\left(Z_{2}, \Omega G\right), \Omega F, G\right), L(F, \Omega G)$. Тогда $\Omega\left(Z_{2}, G\right)$ будет подалгеброй алгебры $\Omega(F, G)$, а $L\left(Z_{2}, \Omega G\right)$ - подалгеброй алгебры $L(F, \Omega G)$. Неравенство $(15)$ верно в алгебре $L\left(Z_{2}, G\right)$. Поэтому оно верно и в алгебpe $L(F, G)$, а следовательно, и в алгебре $\Omega(F, G)$. Наконец, из определения последней алгебры следует, что $\Omega(F, G) \subseteq \omega G$, где $\omega G$ - фундаментальный идеал групповой алгебры $F G$. Имеем $G \subseteq T_{n}$, тогда по п. 1) леммы $2 \omega G \subseteq \omega T_{n}$, т.е. $\Omega(F, G) \subseteq \omega T_{n}$. Следовательно, неравенство $(15)$ верно и в фундаментальном идеале $\omega T_{n}$ групповой алгебры $F T_{n}$. Лемма доказана.

Из лемм 6 и 7 непосредственно получаем следующую теорему.

ТЕоремА 1. В многообразии $\mathfrak{B}$ ассочиативных алгебр над бесконечным полем характеристики 2 система тождеств $M=\left\{\mu_{k}=0 \mid k=1,2, \ldots\right\}$ независима.

Различные подмножества из $M$ определяют различные многообразия, тогда из теоремы 1 вытекает

СлЕДСТВИЕ 1. Многообразие $\mathfrak{B}$ содержит континуум различных не конечно базируемых подмногообразий.

Из леммы 3 следует, что все эти подмногообразия локально нильпотентны и локально конечны.

СлЕДСТВИЕ 2. В многообразии $\mathfrak{B}$ существует алгебра, определенная перечислимым мнохсеством тохдественных соотношений, в которой не разрешима проблема равенства слов.

ДокАЗАТЕЛьство. Пусть $S$ есть некоторое перечислимое и не разрешимое множество чисел. Рассмотрим алгебру $A$ многообразия $\mathfrak{B}$, определенную тождественными соотношениями $\left\{\mu_{n}=0\right\}$ при $n \in S$. Очевидно, каждое соотношение алгебры $A$ есть тождественное соотношение. В силу теоремы 1 произвольное тождество из $\left\{\mu_{n}=0\right\}$ 
при данном $n$ вьполнено в $A$ в том и только том случае, если $n \in S$. Следовательно, в $A$ не разрешима проблема равенства слов.

Известно, что если на аддитивном $F$-модуле $\omega T_{n}$ ввести умножение $\cdot: x \cdot y=x y+y x$, то полученная алгебра будет специальной йордановой, и так как $F$ - поле характеристики 2 , то она будет и лиевой. Тогда из теоремы 1 получаем

СЛЕДСТВИЕ 3. В многообразии $\mathfrak{C}$ (соответственно $\mathfrak{D}$ ) специальных йордановых (соответственно лиевых) алгебр над бесконечным полем характеристики 2 система тождеств $\left\{\nu_{k}=0 \mid k=1,2, \ldots\right\}$ независима.

Как и в случае следствий 1,2 отсюда следует

СЛЕДСТВИЕ 4. Многообразие $\mathfrak{C}$ (соответственно $\mathfrak{D})$ содер жит континуум различных не конечно базируемых подмногообразий, и в $\mathfrak{C}$ (соответственно $\mathfrak{D})$ имеются алгебры, заданные перечислимым множеством тохдественных соотношений, в которых не разрешима проблема равенства слов.

Отметим, что в случае лиевых алгебр следствие 3 не претендует на новизну. Бесконечные системы тождеств для лиевых алгебр над полем характеристики 2 указаны в [13], [14].

Отметим также, что при существенном использовании результатов работы [15] бесконечные системы тождеств ассоциативной алгебры над полем характеристики $p>0$ другими, комбинаторными методами построены также Беловым [16].

\section{СПИСОК ЦИТИРОВАННОЙ ЛИТЕРАТУРЫ}

[1] Specht W. Gesetze in Ringen 1 // Math. Z. 1950. V. 52. P. 557-589.

[2] Днестровская тетрадь. Новосибирск, 1986.

[3] Кемер А. Р. Конечная базируемость тождеств ассоциативных алгебр // Алгебра и логика. 1987. T. 26. № 5. C. 597-641.

[4] Бахтурин Ю.А., Ольшанский А. Ю. Тождества // Итоги науки и техники. Современные проблемы математики. Фундаментальные направления. Т. 18. М.: ВИНИТИ, 1987. C. $117-240$.

[5] Коуровская тетрадь. Новосибирск, 1990.

[6] Залесский А.Е., Михалев А.В. Групповые кольца // Итоги науки и техники. Современные проблемы математики. Фундаментальные направления. Т. 2. М.: ВИНИТИ, 1973. C. $5-118$.

[7] Бовди А. А. Групповые кольца. Ужгород, 1974.

[8] Vaughan-Lee M. R. Uncountably many varieties of groups // Bull. London Math. Soc. 1970. № 2. P. 280-286.

[9] Ленг С. Алгебра. М.: Мир, 1968.

[10] Магнус В., Каррас А., Солитэр Д. Комбинаторная теория групп. М.: Наука, 1974.

[11] Джекобсон Н. Алгебры Ли. М.: Мир, 1964.

[12] Ширшов А. И. Свободные лиевы кольца // Матем. сб. 1958. Т. 45 (87). С. 113-122.

[13] Vaughan-Lee M. R. Varieties of Lie algebras // Quart. J. Math. 1970. V. 21. № 83. P. 297-308.

[14] Vaughan-Lee M. R. Abelian-by-nilpotent varieties of Lie algebras // J. London Math. Soc. 1975. V. 11. № 3. P. 263-266.

[15] Шиголев В. В. Примеры бесконечно базируемых T-пространств // Матем. сб. 2000. T. 191. № 3. C. 143-160.

[16] Белов А. Я. Контрпримеры к проблеме Шпехта // Матем. сб. 2000. Т. 191. № 3. С. 13-24.

Государственный аграрный университет Молдовы,

г. Кишинев 